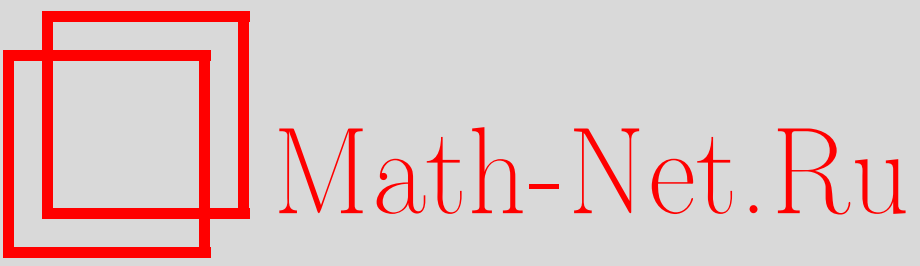

М. А. Соловьев, Лоренц-ковариантные ультрараспределения, гиперфункции и аналитические функционалы, ТМФ, 2001, том 128, номер 3, 492-514

DOI: https://doi.org/10.4213/tmf511

Использование Общероссийского математического портала Math-Net.Ru подразумевает, что вы прочитали и согласны с пользовательским соглашением

http://www.mathnet.ru/rus/agreement

Параметры загрузки:

IP: 3.85 .73 .92

26 апреля 2023 г., 14:58:20 
ТЕОРЕТИЧЕСКАЯ

И МАТЕМАТИЧЕСКАЯ

ФИЗИКА

Том 128, № 3

сентябрь, 2001

(C) 2001 г.

М. А. Соловьев*

\section{ЛОРЕНЦ-КОВАРИАНТНЫЕ УЛЬТРАРАСПРЕДЕЛЕНИЯ, ГИПЕРФУНКЦИИ И АНАЛИТИЧЕСКИЕ ФУНКЦИОНАЛЫ}

Теория лоренц-ковариантных распределений обобщается на более широкие классы функционалов, включая ультрараспределения, гиперфункции и аналитические функционалы умеренного роста. Доказано, что при сколь угодно высокой сингулярности возможно разложение лоренц-ковариантных функционалов по полиномиальным ковариантам, и установлена возможность инвариантного разложения их несущих конусов. Описаны свойства нечетных высокосингулярных обобщенных функций. Полученные результаты применяются к исследованию вакуумных средних нелокальных квантовых полей с произвольным высокоэнергетическим поведением и к обобщению на нелокальную теорию поля теоремы о связи спина со статистикой.

\section{1. ВВЕДЕНИЕ}

Целью данной работы является распространение на функционалы произвольно высокой сингулярности теории лоренц-ковариантных распределений, которая играет важную роль в аксиоматическом подходе [1], [2] в квантовой теории поля (КТП). Основным достижением этого подхода по праву считается вывод теорем о связи спина со статистикой и $P C T$-симметрии. Мой интерес к высокосингулярным квантовым полям возник под влиянием профессора В.Я. Файнберга более 30 лет назад, когда я был его аспирантом. Энтузиазм тех лет, вызванный работами Меймана [3] и Джаффе [4], был основан на надежде получить на этом пути решение проблем неперенормируемых взаимодействий и нуль-заряда и построить последовательную нелокальную теорию поля. Последующее развитие калибровочной теории и теории суперструн показали, что некоторые из выдвинутых тогда идей не утратили своего значения и сейчас. В работах Файнберга и Иофы [5], [6] впервые была предложена формулировка нелокальной теории поля на строгом уровне аксиоматического подхода и продемонстрировано несоблюдение теоремы о глобальной природе локальной коммутативности при экспоненциальном и более быстром росте матричных элементов полей в импульсном пространстве. Именно такой рост, с показателем экспоненты, пропорциональным планковской длине, был обнаружен впоследствии у спектральных плотностей в представлении типа Челлена-Лемана для струнных

\footnotetext{
* Физический институт им П. Н. Лебедева РАН, Москва, Россия. E-mail:soloviev@td.lpi.ac.ru
} 
пропагаторов [7]. Ограничения на амплитуды рассеяния частиц с нелокальным взаимодействием [8]-[10] представляют особый интерес в связи с активно исследуемой сейчас $\mathrm{AdS} / \mathrm{CFT}$ дуальностью (т.е. соответствием между теориями гравитации в пространстве анти-де Ситтера и конформными теориями поля на его границе). При нахождении матрицы рассеяния из корреляторов конформной теории в плоском пределе [11] эти ограничения могут указывать на наличие нелокальности. Использование пропагаторов с нелокальными формфакторами, подавляюшими ультрафиолетовые расходимости в евклидовом импульсном пространстве, оказалось эффективным в лагранжевой формулировке нелокальной КТП [12] и при феноменологическом описании сильных взаимодействий [13]. Имеются развитые схемы такого рода, включаюшие квантовую гравитацию и предлагаемые как феноменологическая альтернатива теории струн [14]. В работе [15] было показано, что наиболее широкие рамки для построения локальной КТП дает использование обобшенных функций, определенных на фурье-симметричном пространстве пробных функций $S_{1}^{1}$, и соответствуюшее обобщение микропричинности. Позже такая формулировка была детально развита [16], [17] в терминах фурье-гиперфункций. При этом оказалось, что она устанавливает полностью симметричное соотношение между КТП в пространстве Минковского и евклидовой теорией поля, чего не удается достичь при использовании обычных распределений умеренного роста. Построенная тогда теория [18] преобразования Фурье-Лапласа функционалов над пространствами $S_{\alpha}^{\beta}$ Гельфанда-Шилова оказалась полезной при операторной реализации калибровочных моделей с индефинитной метрикой [19]-[21], где сингулярности имеют инфракрасное происхождение.

Ряд теорем о высокосингулярных лоренц-ковариантных обобшенных функциях был установлен в работе [22]. Однако там не рассматривались два наиболее интересных и в то же время наиболее трудных для анализа случая - гиперфункций умеренного роста, определенных на пространстве $S^{1}$, универсальном для локальной КТП, и аналитических функционалов над пространством $S^{0}$, на котором определены нелокальные поля с произвольным высокоэнергетическим поведением. В данной статье приведено полное изложение теории для функционалов класса $S^{\prime \beta}, \beta \geqslant 0$. Их использование в теоретико-полевых конструкциях имеет ряд преимуществ, однако топологически пространства пробных функций $S^{\beta}$ устроены сложнее, чем $S_{\alpha}^{\beta}$. Если последние принадлежат к хорошо изученному классу пространств DFS (пространств, дуальных пространствам Фреше-Шварца), то $S^{\beta}$ не входят в него, что затрудняет вывод некоторых структурных теорем для определенных на них функционалов. Эта трудность преодолевается с помощью установленной в разделе 2 теоремы об ацикличности последовательности пространств Фреше, индуктивным пределом которой является $S^{\beta}$. В следуюших трех разделах показана возможность разложения носителей функционалов класса $S^{\prime \beta}$ при $\beta>1$ и $\beta=1$ и их несущих конусов при $\beta<1$, что требует разной аргументации. В разделе 6 эти результаты используются для вывода инвариантных разложений лоренц-ковариантных обобщенных функций. Там же описаны свойства нечетных инвариантных функционалов произвольной сингулярности. В разделе 7 устанавливается плотность ковариантных распределений умеренного роста в рассматриваемых классах ковариантных функционалов, а в разделе 8 на них обобшается представление через полиномиальные ковари- 
анты [2], [23]. В разделе 9 излагается основанный на этих результатах вывод теоремы о связи спина со статистикой для нелокальных квантовых полей, альтернативный выводу работы [24], используюшему понятие аналитического волнового фронта.

\section{2. ПРОСТРАНСТВА $S^{\beta}(O)$ И ИХ ТОПОЛОГИЯ}

По определению [25] пространство пробных функций $S^{\beta}\left(\mathbb{R}^{n}\right)$ с индексом $\beta \geqslant 0$ состоит из бесконечно дифференцируемых функций на $\mathbb{R}^{n}$, удовлетворяюших неравенствам

$$
\left|\partial^{\kappa} f(x)\right| \leqslant C_{N} B^{|\kappa|} \kappa^{\beta \kappa}(1+|x|)^{-N},
$$

где $\kappa$ пробегает множество мультииндексов $\mathbb{Z}^{n}, N$ пробегает множество $\mathbb{N}$ целых неотрицательных чисел, а положительные константы $C_{N}$ и $B$ зависят от функции $f$. Если $\beta>1$, то в пространстве $S^{\beta}$ имеются функции с компактным носителем. Пространство $S^{1}$ состоит из функций, аналитических в комплексной окрестности $\mathbb{R}^{n}$, а при $\beta<1$ составляюшие $S^{\beta}$ функции допускают аналитическое продолжение во все $\mathbb{C}^{n}$, т.е. являются целыми. В первом случае элементы сопряженного пространства $S^{\prime \beta}$ называются ультрараспределениями класса $\left\{\kappa^{\beta \kappa}\right\}$ (умеренного роста), во втором - гиперфункциями, в третьем - аналитическими функционалами (также умеренного роста). Вместо задания топологии в книге [25] была определена сходимость последовательностей в $S^{\beta}$. Именно, неравенствам (1) соответствует норма

$$
\|f\|_{B, N}=\sup _{\kappa, x}(1+|x|)^{N} \frac{\left|\partial^{\kappa} f(x)\right|}{B^{|\kappa|} \kappa^{\beta \kappa}},
$$

и последовательность $f_{\nu} \in S^{\beta}$ считается сходящейся к нулю, если существует такое $B$, что $\left\|f_{\nu}\right\|_{B, N} \rightarrow 0$ при любом $N$. Мы покажем, что это определение полностью согласуется с естественной топологизацией $S^{\beta}$ посредством взятия проективного предела по индексу $N \rightarrow \infty$ и индуктивного по индексу $B \rightarrow \infty$. Другое дополнение теории [25], которое нам также понадобится, состоит в использовании аналогичных пространств над открытыми множествами в $\mathbb{R}^{n}$.

ОПреДЕЛЕНИЕ 1 . Пусть $O$ - непустое открытое множество в $\mathbb{R}^{n}$. Обозначим через $S^{\beta, B, N}(O)$ нормированное пространство бесконечно дифференцируемых функций на $O$, норма $\|f\|_{O, B, N}$ которого задается по аналогии с формулой $(2)$, но операция sup берется по $x \in O$. Через $S^{\beta, B}(O)$ обозначим пересечение $\bigcap_{N} S^{\beta, B, N}(O)$, наделенное проективной топологией, а через $S^{\beta}(O)$ - объединение $\bigcup_{B} S^{\beta, B}(O)$, наделенное индуктивной топологией.

Легко проверить, что пространство $S^{\beta, B, N}(O)$ является полным, т.е. банаховым. Поскольку проективные пределы наследуют свойство полноты, $S^{\beta, B}(O)$ является пространством Фреше. Для доказательства полноты $S^{\beta}(O)$ воспользуемся достаточным условием, указанным Паламодовым [26].

ТЕОРема 1. Инвективная последовательность пространств фреше $S^{\beta, B}(O)$ ащиклична. 
ДокАЗАТЕЛЬСТво. Пусть $\mathcal{U}_{B}$ - окрестность нуля в $S^{\beta, B}(O)$ вида $\|f\|_{O, B, 0}<1 / 2$. Очевидно, $\mathcal{U}_{B_{0}} \subset \mathcal{U}_{B}$ при любом $B>B_{0}$. Согласно теореме 6.1 из работы [26] достаточно убедиться, что топология на $\mathcal{U}_{B_{0}}$, индуцируемая из $S^{\beta, B}(O), B>B_{0}$, не зависит от $B$. Пусть $f_{0} \in \mathcal{U}_{B_{0}}$. Обозначим через $\mathcal{V}_{B, N, \epsilon}$ след на $\mathcal{U}_{B_{0}}$ окрестности функции $f_{0}$ в $S^{\beta, B}(O)$ вида $\left\|f-f_{0}\right\|_{O, B, N}<\epsilon$ и покажем, что для всяких $B>B_{1}>B_{0}$ и любых $N_{1}$, $\epsilon_{1}$ найдутся числа $N, \epsilon$ такие, что $\mathcal{V}_{B, N, \epsilon} \subset \mathcal{V}_{B_{1}, N_{1}, \epsilon_{1}}$. Это будет означать, что топология, индушируемая на $\mathcal{U}_{B_{0}}$ из $S^{\beta, B}(O)$, не слабее, чем индушируемая из $S^{\beta, B_{1}}(O)$; обратное очевидно. Далее для простоты считаем $\beta=0$ (формулы в общем случае отличаются лишь несушественными множителями). Если $f \in \mathcal{V}_{B, N, \epsilon}$, то для функции $f_{1}=f-f_{0}$ имеем две оценки

$$
\left|\partial^{\kappa} f_{1}(x)\right|<B_{0}^{|\kappa|}, \quad\left|\partial^{\kappa} f_{1}(x)\right|<\epsilon B^{|\kappa|}(1+|x|)^{-N} \quad(x \in O) .
$$

Надо показать, что при должном выборе $N$ и $\epsilon$ отсюда следует, что

$$
\left|\partial^{\kappa} f_{1}(x)\right|<\epsilon_{1} B_{1}^{|\kappa|}(1+|x|)^{-N_{1}} \quad(x \in O) .
$$

Введем обозначения $\varepsilon=\epsilon(1+|x|)^{-N}, \varepsilon_{1}=\epsilon_{1}(1+|x|)^{-N_{1}}$ и определим число $Q(x)$ уравнением $B_{0}^{Q}=\varepsilon_{1} B_{1}^{Q}$. Если $x$ фиксирован, то первое из неравенств (3) влечет (4) при всех $|\kappa| \geqslant Q$. Второе из неравенств (3) влечет (4) при $|\kappa|<Q$ в том случае, если $\varepsilon B^{Q} \leqslant$ $\varepsilon_{1} B_{1}^{Q}$. Полагая $\varepsilon B^{Q}=\varepsilon_{1} B_{1}^{Q}$, получаем $\varepsilon=\varepsilon_{1}^{A}$, где число $A=\ln \left(B / B_{0}\right) / \ln \left(B_{1} / B_{0}\right)$ не зависит от $x$. Значит, нужная импликация будет обеспечена, если взять $\epsilon \leqslant \epsilon_{1}^{A}$ и $N \geqslant A N_{1}$, что завершает доказательство.

Согласно [26] ацикличность обеспечивает справедливость следуюших утверждений.

СледСтвИЕ. Пространство $S^{\beta}(O)$ отделимо и полно. Множество $\mathcal{B} \subset S^{\beta}(O)$ является ограниченным в том и только в том случае, если оно челиком содержится в некотором пространстве $S^{\beta, B}(O)$ и ограничено по каждой из его норм.

Отделимость пространства $S^{\beta}(O)$ очевидна, поскольку его топология мажорирует топологию равномерной сходимости. Отметим еше, что индуктивный предел пространств Фреше является борнологическим пространством, поэтому непрерывность линейного отображения $S^{\beta}(O)$ в произвольное локально-выпуклое пространство равносильна его ограниченности на всех ограниченных множествах, что, в свою очередь, эквивалентно секвенциальной непрерывности [27]. Роль пространств $S^{\beta}(O)$ в вопросах локализации ясна из следуюшего простого замечания. Ультрараспределение $v \in S^{\prime \beta}$ имеет носитель в компакте $K$ в том и только в том случае, если для любой его окрестности $O$ найдется функционал $\hat{v} \in S^{\prime \beta}(O)$ такой, что $(v, f)=\left(\hat{v},\left.f\right|_{O}\right)$ для всех $f \in S^{\beta}$. При $\beta \leqslant 1$, когда среди пробных функций нет финитных, это можно взять за основу определения понятия сосредоточенности.

В книге [28] доказана ядерность пространств $S^{\beta, B+}=\bigcap_{\epsilon>0} S^{\beta, B+\epsilon}$ (точнее, фурьеизоморфных им пространств $\left.S_{\beta, B+}\right)$. Отсюда следует ядерность пространства $S^{\beta}$, поскольку индуктивные пределы счетных семейств пространств наследуют это важное свойство ([27], § III.7.4). В свою очередь, ядерность пространства вместе с его полнотой приводят к тому, что оно является монтелевским и, в частности, рефлексивным ([27], гл. IV, упр. 19). Мы будем использовать эти свойства $S^{\beta}\left(\mathbb{R}^{n}\right)$ в дальнейшем. Пространства $S^{\beta}(O)$ обладают ими лишь при некоторых ограничениях на $O$. 


\section{3. РАЗЛОЖЕНИЕ УЛЬТРАРАСПРЕДЕЛЕНИЙ}

Пусть $v$ - распределение умеренного роста, определенное на пространстве Шварца $S$, и пусть носитель $v$ содержится в объединении замкнутых множеств $K_{1}$ и $K_{2}$. Как известно, разложение вида $v=v_{1}+v_{2}$ для распределений $v_{1,2} \in S^{\prime}$ с носителями в $K_{1,2}$ возможно в том случае, если эти множества достаточно регулярны и регулярно расположены друг относительно друга. Точная формулировка условий регулярности устанавливается с помощью теоремы продолжения Уитни [29]. Эти условия заведомо выполнены для множеств, представимых в виде объединения конечного числа замкнутых выпуклых подмножеств с непустой внутренностью. Для дальнейшего полезно напомнить вьвод теоремы разложения в этом простейшем (однако достаточном для большинства приложений) случае. Пусть $K$ - множество указанного вида в $\mathbb{R}^{n}$. Обозначим через $S(K)$ пространство бесконечно дифференцируемых функций на его внутренности Int $K$, для которых продолжения производных по непрерывности на гранишы выпуклых составляюших совпадают друг с другом, когда границы имеют общие точки, и таких, что нормы

$$
\max _{|\kappa| \leqslant N} \sup _{x \in K}(1+|x|)^{N}\left|\partial^{\kappa} f(x)\right|
$$

конечны. Это пространство относится к классу пространств FS (Фреше-Шварца). Согласно теореме Уитни возможно дальнейшее продолжение $f$ до гладкой функции на $\mathbb{R}^{n}$, и из нее вытекает, что пространство распределений Шварца с носителем в $K$ можно отождествить с сопряженным к $S(K)$ пространством $S^{\prime}(K)$. Каноническое отображение

$$
S\left(K_{1} \cup K_{2}\right) \longrightarrow S\left(K_{1}\right) \oplus S\left(K_{2}\right)
$$

инъективно, непрерывно и имеет замкнутый образ, так как совпадение сходящихся последовательностей $f_{1 \nu}, f_{2 \nu}$ на пересечении $K_{1} \cap K_{2}$ влечет совпадение на нем их пределов, которые тем самым определяют некоторый элемент пространства $S\left(K_{1} \cup K_{2}\right)$. Таким образом, это пространство можно рассматривать как подпространство суммы $S\left(K_{1}\right) \oplus S\left(K_{2}\right)$. Такое отождествление справедливо не только алгебраически, но и топологически, поскольку замкнутое подпространство суммы пространств FS также является пространством FS, а согласно теореме об открытом отображении [27] векторное пространство не может иметь двух разных сравнимых топологий, в каждой из которых оно является пространством Фреше. Применяя теорему Хана-Банаха, заключаем, что любой функционал $v \in S^{\prime}\left(K_{1} \cup K_{2}\right)$ имеет непрерывное продолжение на сумму. Обозначая его $\hat{v}$ и записывая $v(f)=\hat{v}\left(\left.f\right|_{K_{1}}, 0\right)+\hat{v}\left(0,\left.f\right|_{K_{2}}\right)$, получаем желаемое разложение, поскольку композиция $\hat{v}$ с каноническими вложениями $S\left(K_{i}\right) \rightarrow S\left(K_{1}\right) \oplus \mathcal{S}\left(K_{2}\right)$ дает элементы $S^{\prime}\left(K_{i}\right)(i=1,2)$.

Условия разложимости ультрараспределений изучались Ламбертом [30]. Полученная им теорема охватывает, в частности, функционалы класса $S_{0}^{\prime \beta}$ Гельфанда-Шилова. (Напомним, что пространство $S_{0}^{\beta}$ состоит из тех элементов $S^{\beta}$, которые имеют компактньй носитель, и нетривиально при $\beta>1$.) Вывод аналогичной теоремы для класса $S^{\prime} \beta$ затруднителен, но в том частном случае, который только и важен для интересующих нас лоренц-инвариантных разложений, рассматриваемых ниже, соответствующее утверждение является прямым следствием результатов Ламберта. 
ТЕОРема 2. Пусть $K_{1}, K_{2}$ - замкнутые выпуклье конусы в $\mathbb{R}^{n}$ такие, что $K_{1} \cap K_{2}=\{0\}$. Любой функиионал $v \in S^{\prime \beta}$ с носителем в конусе $K_{1} \cup K_{2}$ допускает разложсение в сумму функиионалов того жсе класса $S^{\prime \beta}$ с носителями в конусах $K_{1}$ и $K_{2}$.

ДокАЗАТЕльство. Согласно теореме V.1.1 из работы [30] сужение $\left.v\right|_{S_{0}^{\beta}}$ представимо в виде суммы $v_{1}+v_{2}$, где функционалы $v_{1}, v_{2}$ имеют носители в $K_{1}$ и $K_{2}$, соответственно. Надо убедиться лишь в том, что они имеют непрерывное продолжение на $S^{\beta}$, которое с необходимостью единственно, ибо $S_{0}^{\beta}$ плотно в $S^{\beta}$. Пусть $\chi$ - любая функция из $S_{0}^{\beta}$, тождественно равная 1 на шаре $U=\{x:|x|<1\}$, и пусть $\chi_{1,2}$ - мультипликаторы в $S^{\beta}$ (а значит, и в $S_{0}^{\beta}$ ), равные 1 в окрестности $K_{1,2} \backslash U$ и нулю в окрестности $K_{2,1} \backslash U$. Поскольку $S_{0}^{\beta}$ является алгеброй по умножению, при всех $f \in S_{0}^{\beta}$ имеем равенство

$$
v_{1,2}(f)=v_{1,2}(\chi f)+v\left(\chi_{1,2}(1-\chi) f\right)
$$

Остается заметить, что умножение на $\chi$ непрерывно отображает $S^{\beta}$ в $S_{0}^{\beta}$ и, следовательно, правая часть в (7) определена как непрерывный функционал на $S^{\beta}$.

\section{4. РАЗЛОЖЕНИЕ ГИПЕРФУНКЦИЙ}

Установленные в [30] условия регулярности множеств, гарантирующие возможность разложения, оказываются все более слабыми по мере увеличения сингулярности функционалов, т.е. по мере уменьшения индекса $\beta$ пространства пробных функций. В классе $S^{\prime 1}$ разложение функционала с носителем в $K_{1} \cup K_{2}$ возможно уже для любых компактных множеств $K_{1}, K_{2}$. Однако само определение носителя в данном случае иное, поскольку пробные функции аналитичны. Обозначим через $A(K)$ пространство функций, аналитических в комплексной окрестности (зависящей от функции) компакта $K \subset \mathbb{R}^{n}$ и напомним, что при наделении естественной топологией оно становится пространством DFS [31]. Прямое применение формулы Тейлора показывает, что $S^{1}$ непрерывно вложено в $A(K)$. В соответствии со стандартным определением [29], [31] несушего множества аналитического функционала компакт $K$ является таковым для $v \in S^{\prime 1}$, если $v$ допускает непрерывное продолжение на $A(K)$. Это можно еше выразить формулой $v \in A^{\prime}(K)$, поскольку $S^{1}$ плотно в $A(K)$. Теорема разложения для $v \in A^{\prime}\left(K_{1} \cup K_{2}\right)$ легко устанавливается тем же рассуждением, что и в предыдушем разделе, так как замкнутое подпространство суммы пространств DFS является пространством DFS и к пространствам этого класса также применима теорема об открытом отображении. Другое доказательство, использующее гармоническую регуляризацию аналитических функционалов, дано в книге [29] (§9.1).

При формализации понятия носителя элементов $v \in S^{\prime 1}$ необходимо учитывать, что некоторые из них естественно считать сосредоточенными на бесконечности. Дело в том, что индуктивный предел $\lim _{\longrightarrow} S^{1}\left(O_{R}\right)$, где $O_{R}=\{x:|x|>R\}$ и $R \rightarrow \infty$, является отделимым (хаусдорфовым) пространством, в которое $S^{1}$ инъективно и непрерывно вложено (см. предложение 1.19 в статье [22]). Поэтому согласно теореме Хана-Банаха в $S^{\prime 1}$ сушествуют ненулевые функционалы, допускаюшие непрерывное продолжение на это 
пространство. В теории фурье-гиперфункций [32] применяется радиальная компактификация $\mathbb{D}^{n}=\mathbb{R}^{n} \sqcup \mathbb{S}_{\infty}^{n-1}$, где $\mathbb{S}_{\infty}^{n-1}$ - бесконечно удаленная $(n-1)$-мерная сфера. Мы воспользуемся ею и в случае функционалов класса $S^{\prime 1}$. Считаем компакт $\mathcal{K} \in \mathbb{D}^{n}$ несущим для $v \in S^{\prime}$, если $v$ допускает непрерывное продолжение на пространство

$$
S^{1}(\mathcal{K})=\underset{\mathcal{O} \supset \mathcal{K}}{\lim _{\longrightarrow}} S^{1}\left(\mathcal{O} \cap \mathbb{R}^{n}\right)
$$

где $\mathcal{O}$ пробегает открытые окрестности $\mathcal{K}$ в $\mathbb{D}^{n}$. Если $\mathcal{K} \subset \mathbb{R}^{n}$, то это определение сводится к предыдушему, ибо тогда индуктивный предел (8) совпадает с $A(\mathcal{K})$. Напомним, что фурье-гиперфункции формируют пространство, сопряженное пространству пробных функций $S_{1}^{1}$, определяемому неравенствами

$$
\left|\partial^{\kappa} f(x)\right| \leqslant C B^{|\kappa|} \kappa^{\beta \kappa} \exp \left(-\left|\frac{x}{A}\right|\right)
$$

где константы $A, B, C$ зависят от $f$. Пространства $S_{1}^{1}(O)$ представляют собой объединения банаховых пространств $S_{1, A}^{1, B}(O)$, связанных компактными вложениями, по обоим индексам $A, B$. Поэтому и $S_{1}^{1}(O)$, и $S_{1}^{1}(\mathcal{K})$ являются пространствами DFS. Рассматривая каноническое отображение

$$
S_{1}^{1}\left(\mathcal{K}_{1} \cup \mathcal{K}_{2}\right) \longrightarrow S_{1}^{1}\left(\mathcal{K}_{1}\right) \oplus S_{1}^{1}\left(\mathcal{K}_{2}\right)
$$

и используя все то же простое рассуждение предыдушего раздела, мы заключаем, что разложение фурье-гиперфункций с носителем в $\mathcal{K}_{1} \cup \mathcal{K}_{2}$ возможно для любых компактов $\mathcal{K}_{1}, \mathcal{K}_{2} \subset \mathbb{D}^{n}$.

Единственное препятствие для аналогичного вывода теоремы разложения в случае функционалов класса $S^{\prime 1}$ состоит в том, что становится неочевидной возможность применения теоремы об открытом отображении. Ее наиболее общая формулировка [33] предполагает, что пространство значений отображения является ультраборнологическим, т.е. отделимым индуктивным пределом пространств Фреше. Вовсе не очевидно, что $S^{1}(\mathcal{K}), \mathcal{K}=\mathcal{K}_{1} \cup \mathcal{K}_{2}$, входит в этот класс при наделении его топологией, индуцируемой вложением в сумму $S^{1}\left(\mathcal{K}_{1}\right) \oplus S^{1}\left(\mathcal{K}_{2}\right)$. Чтобы глубже понять ситуацию, следует рассмотреть цепочку отображений

$$
0 \longrightarrow S^{1}\left(\mathcal{K}_{1} \cup \mathcal{K}_{2}\right) \stackrel{i}{\longrightarrow} S^{1}\left(\mathcal{K}_{1}\right) \oplus S^{1}\left(\mathcal{K}_{2}\right) \stackrel{s}{\longrightarrow} S^{1}\left(\mathcal{K}_{1} \cap \mathcal{K}_{2}\right) \longrightarrow 0
$$

где отображение $s$ сопоставляет паре функций $f_{1,2} \in S^{1}\left(\mathcal{K}_{1,2}\right)$ разность их ограничений на $\mathcal{K}_{1} \cap \mathcal{K}_{2}$. Теорема 1.30 из статьи [22] устанавливает, что последовательность векторных пространств (11) является точной, т.е. ядро каждого из участвующих отображений совпадает с образом предыдушего. Нетривиальна лишш точность в члене $S^{1}\left(\mathcal{K}_{1} \cap \mathcal{K}_{2}\right)$, которая означает, что любой элемент этого пространства допускает разложение на функции из пространств $S^{1}\left(\mathcal{K}_{1}\right)$ и $S^{1}\left(\mathcal{K}_{2}\right)$, что устанавливается с помошью $L^{2}$-оценок Хёрмандера, как и аналогичное утверждение в случае фурье-гиперфункций. Из этого результата вытекает сушествование носителей у функционалов класса $S^{\prime 1}\left(\mathbb{R}^{n}\right)$. 
ТЕОРемА 3. Каждый әлемент пространства $S^{\prime 1}\left(\mathbb{R}^{n}\right)$ имеет единственньй минимальный несущий компакт в $\mathbb{D}^{n}$.

ДокАЗАТЕЛЬСтво. Прежде всего отметим, что если у функционала $v \in S^{\prime 1}\left(\mathbb{R}^{n}\right)$ имеются несущие компакты $\mathcal{K}_{1}, \mathcal{K}_{2}$ с пустым пересечением, то $v=0$. Это следует из плотности $S^{1}$ в $S^{1}\left(\mathcal{K}_{1} \cup \mathcal{K}_{2}\right.$ ) (устанавливаемой леммой 1.17 в [22]), ибо тогда это пространство содержит функцию, тождественно равную нулю в окрестности $\mathcal{K}_{1}$ и равную любому заданному элементу $S^{1}$ в окрестности $\mathcal{K}_{2}$. Пусть теперь несущие компакты имеют непустое пересечение $\mathcal{K}_{1} \cap \mathcal{K}_{2}$. Для доказательства теоремы достаточно показать, что и оно будет несущим множеством для $v$. Действительно, предположим, что это так, и обозначим через $\mathcal{K}$ пересечение всех несущих компактов $v$. Если найдется конечная подсистема несуших множеств с пустым пересечением, то $v=0$. Если такой подсистемы нет, то $\mathcal{K} \neq \varnothing$. Пусть $\mathcal{O}$ - окрестность $\mathcal{K}$ в $\mathbb{D}^{n}$. Дополнения несуших компактов образуют открытое покрытие компакта $\mathbb{D}^{n} \backslash \mathcal{O}$. Выбирая из него конечное подпокрытие, заключаем, что $\mathcal{K}$ - минимальный несуший компакт.

Переходя к ортогональным дополнениям в равенстве $\operatorname{Im} i=\operatorname{Ker} s$, мы получаем Ker $i^{\prime}=\overline{\operatorname{Im} s^{\prime}}$, где штрихами отмечены сопряженные отображения, а черта означает замыкание в слабой топологии. К отображению $s$ в (11) применима теорема об открытом отображении в формулировке Гротендика [34], поскольку оно отображает пространство класса $\mathcal{U F}^{1)}$ в ультраборнологическое пространство. В частности, $s$ является топологическим гомоморфизмом. Следовательно, образ отображения $s^{\prime}$ слабо замкнут по теореме IV .7.5 из книги [27] и $\operatorname{Ker} i^{\prime}=\operatorname{Im} s^{\prime}$. Если $v \in S^{\prime 1}$ имеет непрерывные продолжения $v_{1,2}$ на $S^{1}\left(\mathcal{K}_{1,2}\right)$, то $\left.i^{\prime}\left(v_{1}, v_{2}\right)\right|_{S^{1}}=0$. Ввиду плотности $S^{1}$ в $S^{1}\left(\mathcal{K}_{1} \cup \mathcal{K}_{2}\right)$ это влечет $i^{\prime}\left(v_{1}, v_{2}\right)=0$. Значит, функционалы $v_{1,2}$ представляют собой ограничения на $S^{1}\left(\mathcal{K}_{1,2}\right)$ некоторого элемента из $S^{\prime 1}\left(\mathcal{K}_{1} \cap \mathcal{K}_{2}\right)$, т.е. сушествует непрерывное продолжение $v$ на $S^{1}\left(\mathcal{K}_{1} \cap \mathcal{K}_{2}\right)$, что завершает доказательство.

ТЕОрема 4. Пусть $\mathcal{K}_{1}, \mathcal{K}_{2}$ - компакты в $\mathbb{D}^{n}$, являющиеся замыканиями конусов и такие, что $\mathcal{K}_{1} \cap \mathcal{K}_{2}=\{0\}$. Любой функционал $v \in S^{\prime 1}$ с носителем в $\mathcal{K}_{1} \cup \mathcal{K}_{2}$ допускает разложсение в сумму функционалов того же класса с носителями в компактах $\mathcal{K}_{1}$ u $\mathcal{K}_{2}$.

Доказательство. Обозначим пространство $S^{1}\left(\mathcal{K}_{1}\right) \oplus S^{1}\left(\mathcal{K}_{2}\right)$ через $E$. Пусть $\mathcal{O}_{1,2}^{\nu}(\nu=1,2, \ldots)$ - счетные фундаментальные системы окрестностей $\mathcal{K}_{1,2}$ в $\mathbb{D}^{n}$. Топология $E$ тождественна топологии индуктивного предела последовательности пространств Фреше $E_{\nu}=S^{1, \nu}\left(\mathcal{O}_{1}^{\nu} \cap \mathbb{R}^{n}\right) \oplus S^{1, \nu}\left(\mathcal{O}_{2}^{\nu} \cap \mathbb{R}^{n}\right)$. Пусть $L$ - образ пространства $S^{1}\left(\mathcal{K}_{1} \cup \mathcal{K}_{2}\right)$ в $E$, снабженный индуцируемой из $E$ топологией. Достаточно показать, что она совпадает с топологией индуктивного предела семейства подпространств $L_{\nu}=E_{\nu} \cap L . \quad$ Действительно, тогда пространство $L$ является ультраборнологическим (поскольку замкнутость $L$ в $E$ влечет замкнутость $L_{\nu}$ в $E_{\nu}$, т.е. каждое

\footnotetext{
1) К этому классу относятся отделимые локально выпуклые пространства, покрываемые счетным семейством своих подпространств $\Phi$ реше. Любое $S^{1}(\mathcal{K})$ входит в него, ибо $S^{1, B}\left(\mathcal{O} \cap \mathbb{R}^{n}\right)$, $\mathcal{O} \supset \mathcal{K}$, являются его подпространствами $\Phi$ реше и $\mathcal{K}$ имеет счетную фундаментальную систему окрестностей в $\mathbb{D}^{n}$.
} 
$L_{\nu}$ есть пространство Фреше), что позволяет применить теорему Гротендика [34] и завершить доказательство с помошью прежней аргументации. Условие совпадения двух указанных топологий на $L$ устанавливается теоремой Ретаха [35] и состоит в ацикличности последовательности фактор-пространств $F_{\nu}=E_{\nu} / L_{\nu}$, которые также являются пространствами Фреше и связаны каноническими инъекциями $F_{\nu} \rightarrow F_{\mu}, \nu<\mu$. Подпространство $L_{\nu}$ служит ядром непрерывного отображения $E_{\nu} \rightarrow E / L$, поэтому соответствуюшее отображение $E_{\nu} / L_{\nu} \rightarrow E / L$ также непрерьвно. Фактор-пространство $E / L$, снабженное индуктивной топологией относительно этого семейства отображений (которая на самом деле совпадает с его собственной), обозначим через $F$. Из точности последовательности (11) следует, что непрерьвное отображение $F \rightarrow S^{1}(\{0\})$ биективно. Пространство $S^{1}(\{0\})$ совпадает с кольцом ростков аналитических функций в точке $z=0$ и принадлежит классу DFS, следовательно, эта биекция является не только алгебраическим, но и топологическим изоморфизмом по теореме из монографии [34]. Посредством обозначения $a_{\kappa}=\partial^{\kappa} f(0)$ пространство $S^{1}(\{0\})$ отождествляется с пространством строк $\left\{a_{\kappa}\right\}$ комплексных чисел таких, что $\left|a_{\kappa}\right| \leqslant C B^{|\kappa|} \kappa^{\kappa}$ при некоторых $C, B>0$. Пусть $A_{\nu}$ - банахово пространство строк с нормой $\sup _{\kappa}\left|a_{\kappa}\right| / \nu^{|\kappa|} \kappa^{\kappa}$. Доказательство теоремы 1 (где в данном простейшем случае надо положить $x=0$ ) показывает, что инъективная последовательность пространств $A_{\nu}$ ациклична. Значит, и последовательность $F_{\nu}$ ациклична, ибо эквивалентна последовательности $A_{\nu}$ ввиду указанного изоморфизма. Тем самым теорема 4 доказана.

\section{5. РАЗЛОЖЕНИЕ ФУНКЦИОНАЛОВ КЛАССА $S^{\prime \beta}, \beta<1$}

В работах [22], [36] показано, что аналитические функционалы класса $S^{\prime \beta}$ (и $S_{\alpha}^{\prime \beta}$ ), $0<\beta<1$, сохраняют угловую локализуемость, хотя и не имеют носителей. Именно, если вместо произвольных компактов в $\mathbb{D}^{n}$ рассматривать лишш замыкания конусов и определить $S^{\beta}(\mathcal{K})$ по аналогии с формулой $(8)$, то последовательность

$$
0 \longrightarrow S^{\beta}\left(\mathcal{K}_{1} \cup \mathcal{K}_{2}\right) \longrightarrow S^{\beta}\left(\mathcal{K}_{1}\right) \oplus S^{\beta}\left(\mathcal{K}_{2}\right) \longrightarrow S^{\beta}\left(\mathcal{K}_{1} \cap \mathcal{K}_{2}\right) \longrightarrow 0
$$

оказывается точной, откуда следует сушествование у каждого элемента $S^{\prime \beta}$ наименьшего замкнутого несушего конуса (мы называем замкнутый конус в $\mathbb{R}^{n}$ несушим, если несушим множеством является компакт, получаемый присоединением к этому конусу вырезаемой им части нароста $\left.\mathbb{S}_{\infty}^{n-1}\right)$. Как и в случае гиперфункций, вывод этого результата основан на использовании представления ассоциированных с конусами пространств $S^{\beta}(\mathcal{K})$ в терминах комплексных переменных. Именно, пусть $O$ - объединение открытого конуса $U \subset \mathbb{R}^{n}$ с $\epsilon$-окрестностью начала координат. Как показано в статье [36], пространство $S^{\beta}(O)$ изоморфно пространству целых функций на $\mathbb{C}^{n}$, удовлетворяющих неравенствам

$$
|f(z)| \leqslant C_{N}(1+|x|)^{-N} \exp \left[|B y|^{\frac{1}{1-\beta}}+d(B x, U)^{\frac{1}{1-\beta}}\right], \quad z=x+i y,
$$

где константы $C_{N}$ и $B$ зависят от $f$ и $d(\cdot, U)$ есть расстояние от точки до конуса $U$. Если $\mathcal{K}$ - компакт в $\mathbb{D}^{n}$, соответствуюший замкнутому конусу $K$, то каждый элемент 
$S^{\beta}(\mathcal{K})$ принадлежит некоторому пространству $S^{\beta}(O)$, где $O$ имеет указанный вид и содержит $K$. Пространство $S^{\beta}(\{0\})$, ассоциированное с вырожденным конусом $\{0\}$, состоит из целых функций с ростом не выше порядка $1 /(1-\beta)$ и конечного типа, т.е. удовлетворяюших ограничению

$$
|f(z)| \leqslant C \exp \left(|B x|^{\frac{1}{1-\beta}}+|B y|^{\frac{1}{1-\beta}}\right) .
$$

ТеОРема 5. Пусть $K_{1}, K_{2}$ - замкнутые конусы в $\mathbb{R}^{n}$ такие, что $K_{1} \cap K_{2}=\{0\}$. Любой функционал $v \in S^{\prime \beta}, \quad 0 \leqslant \beta<1$, с несущим конусом $K_{1} \cup K_{2}$ допускает разложение в сумму функиионалов того же класса с несущими конусами $K_{1}$ и $K_{2}$.

ДоКАЗАТЕЛЬСТво. Утверждение теоремы выводится из точности последовательности (12) рассуждением, полностью аналогичным доказательству теоремы 4 , поскольку $S^{\beta}(\{0\})$ является инъективным пределом ациклической последовательности банаховых пространств, как и $S^{1}(\{0\})$. В основе лежит возможность разложения каждого элемента $S^{\beta}\left(K_{1} \cap K_{2}\right)$ на функции из $S^{\beta}\left(K_{1}\right)$ и $S^{\beta}\left(K_{2}\right)$. Стоит отметить, что при ненулевом $\beta$ для данной геометрии такая возможность практически очевидна, если учесть, что пространство $S_{1-\beta}^{\beta}(\beta>0)$ нетривиально [25] и в нем найдется функция $\chi_{0}$ со свойствами

$$
\left|\chi_{0}(z)\right| \leqslant C_{0} \exp \left(-\left|\frac{x}{A_{0}}\right|^{\frac{1}{1-\beta}}+\left|B_{0} y\right|^{\frac{1}{1-\beta}}\right)
$$

и $\int \chi_{0}(\xi) \mathrm{d} \xi=1$. Пусть $W_{1}, W_{2}$ - открытые конусы такие, что $K_{1,2} \backslash\{0\} \subset W_{1,2}$ и $\bar{W}_{1} \cap$ $\bar{W}_{2}=\{0\}$. Тогда при всех $x \in W_{1}, \xi \in W_{2}$ соблюдается неравенство $|x-\xi| \geqslant \theta|x|$, где $\theta>0$. Положим $\chi(z)=\int_{W_{2}} \chi_{0}(z-\xi) \mathrm{d} \xi$. Имеем очевидную оценку

$$
|\chi(z)| \leqslant C_{A} \exp \left(-\left|\frac{\theta x}{A}\right|^{\frac{1}{1-\beta}}+\left|B_{0} y\right|^{\frac{1}{1-\beta}}\right), \quad x \in W_{1}
$$

справедливую при любом $A>A_{0}$. Желаемое разложение функций, удовлетворяющих ограничению (14), реализуется формулой $f=\chi f+(1-\chi) f$, если взять $A_{0}<\theta / B$, что всегда возможно. Действительно, тогда оценка типа (13) (с достаточно большим $B_{1}$ вместо $B$ ) заведомо выполнена для функции $\chi f$ при $x \in W_{1}$, а если конус $U \supset K \backslash\{0\}$ таков, что $\bar{U} \backslash\{0\} \subset W_{1}$, то она выполнена и при $x \notin W_{1}$, ибо тогда $d(x, U) \geqslant \theta^{\prime}|x|$ с некоторым $\theta^{\prime}>0$. Тем самым $\chi f \in S^{\beta}\left(K_{1}\right)$. Аналогичным образом $(1-\chi) f \in S^{\beta}\left(K_{2}\right)$, ибо $1-\chi=\int_{C W_{2}} \chi_{0}(z-\xi) \mathrm{d} \xi$ и конус $C W_{2}$ отделен от $K_{2}$ конечным угловым расстоянием. При $\beta=0$ эта аргументация неприменима и приходится использовать более специальные средства, как и в теории гиперфункций. В работе [37] доказана точность аналога последовательности (12) для пространств $S_{\alpha}^{0}, \alpha>1$. В частности, элементы из $S^{0}(\{0\})=S_{\alpha}^{0}(\{0\})$ допускают разложение даже в рамках этого, более узкого класса. Таким образом, теорема 5 справедлива и при $\beta=0$. Отметим, что и сама последовательность (12) точна при $\beta=0$, в чем можно убедиться с помошью леммы 1.31 работы [24], но это доказательство значительно сложнее. 
ЛЕмма 1. Любой функиионал $v \in S^{\prime \beta}, \beta \geqslant 0$, несущим множеством которого служит начало координат, имеет вид

$$
v=\sum_{\kappa} c_{\kappa} \partial^{\kappa} \delta(x), \quad \lim _{|\kappa| \rightarrow \infty}|\kappa|^{\beta}\left|c_{\kappa}\right|^{\frac{1}{|\kappa|}}=0 .
$$

ДокАЗАТЕЛЬСТво. При $\beta>1$ это утверждение является простейшим частным случаем теоремы IV.I.I из [30]. Если $\beta \leqslant 1$, то функции $e_{\kappa}=x^{\kappa} / \kappa$ ! очевидно составляют безусловный базис в $S^{\beta}(\{0\})$, а функционалы $e_{\kappa}^{\prime}=(-1)^{|\kappa|} \partial^{\kappa} \delta(x)$ образуют дуальньй ему базис функционалов. Тем самым $(v, f)=\sum_{\kappa}\left(v, e_{\kappa}\right)\left(e_{\kappa}^{\prime}, f\right)$, что переходит в представление (17) после переобозначения $c_{\kappa}=(-1)^{|\kappa|}\left(v, e_{\kappa}\right)$. В силу непрерывности $v$ по топологии $S^{\beta}(\{0\})$ при любом $B$ имеем неравенство $\left|\left(v, e_{\kappa}\right)\right| \leqslant C_{B}\left\|e_{\kappa}\right\|_{B}$, где $\left\|e_{\kappa}\right\|_{B}=\sup _{\ell}\left|\partial^{\ell} e_{\kappa}(0)\right| B^{-|\ell|} \ell^{-\beta \ell}=B^{-|\kappa|} \kappa^{-\beta \kappa}$, откуда следует указанное ограничение на коэффициенты $c_{\kappa}$. Лемма 1 доказана.

\section{6. ЛОРЕНЦ-ИНВАРИАНТНОЕ РАЗЛОЖКНИЕ}

Рассматривая лоренц-инвариантные функционалы на пространстве Минковского $\mathbb{R}^{4}$, будем использовать обозначения $\overline{\mathbb{V}}=\left\{x \in \mathbb{R}^{4}: x^{2}=x_{0} y_{0}-\mathbf{x y} \geqslant 0\right\}, \overline{\mathbb{V}}_{+}=\{x \in \overline{\mathbb{V}}$ : $\left.x_{0} \geqslant 0\right\}$ и $\overline{\mathbb{V}}_{-}=\left\{x \in \overline{\mathbb{V}}: x_{0} \leqslant 0\right\}$. Собственную группу Лоренца обозначим $L_{+}^{\uparrow}$.

ТЕОрема 6. Любой лорени-инвариантный функиионал $v \in S^{\prime \beta}, \beta \geqslant 0$, снесущим конусом $\overline{\mathbb{V}}$ допускает разложсене на лорени-инвариантные функииональ того же класса с несущими конусами $\overline{\mathbb{V}}_{+} u \overline{\mathbb{V}}_{-}$.

ДокАЗАТЕЛЬСТво. Пусть $v=v_{+}-v_{-}-$разложение $v$ на функционалы с несущими верхним и нижнем конусами, сушествуюшее в силу теорем $2,4,5$ (знак минус в нем удобен для дальнейшего). Инвариантность разложения относительно подгруппы пространственных врашений достигается переходом к усредненным функционалам $\bar{v}_{ \pm}$,

$$
\left(\bar{v}_{ \pm}, f\right) \stackrel{\text { def }}{=}\left(v_{ \pm}, \int_{R \in S O(3)} f(R x) d R\right) .
$$

Меру на группе врашений считаем нормированной на $1 . \mathrm{K}$ чисто лоренцевым преобразованиям (бустам) этот прием применить нельзя ввиду их некомпактности. Пусть $\mathrm{N}_{j}=x_{j} \partial_{0}+x_{0} \partial_{j}-$ генераторы представления бустов в пространстве функционалов. Очевидно, что при $\beta>1$ носителем функционала

$$
u=\mathrm{N}_{1} \bar{v}_{+}=\mathrm{N}_{1} \bar{v}_{-}
$$

служит начало координат. При $\beta=1$ такое же утверждение верно по теореме 3 , a при $\beta<1$ вырожденный конус $\{0\}$ является несущим для $v$ ввиду точности последовательности (12) (и лоренц-инвариантности $S^{\prime \beta}\left(\overline{\mathbb{V}}_{ \pm}\right)$). Чтобы получить желаемое разложение, достаточно найти среди функционалов вида (17) $S O(3)$-инвариантное решение уравнения

$$
\mathrm{N}_{1} v=u .
$$


Действительно, если такое решение $v_{0}$ существует, то функционалы $\bar{v}_{ \pm}-v_{0} \in S^{\prime \beta}\left(\overline{\mathbb{V}}_{ \pm}\right)$ инвариантны относительно всей группы $L_{+}^{\uparrow}$ в силу коммутационных соотношений

$$
\left[\mathrm{N}_{j}, \mathrm{M}_{i j}\right]=\mathrm{N}_{i}, \quad i \neq j,
$$

где $\mathrm{M}_{i j}=x_{j} \partial_{i}-x_{i} \partial_{j}$-генераторы представления группы трехмерных врашений. Пусть $\mathrm{C}=-\sum_{i<j} \mathrm{M}_{i j}-$ ее оператор Казимира. Из соотношений (21) следует, что

$$
\mathrm{N}_{1} u=0, \quad \mathrm{C} u=2 u .
$$

Конечномерные пространства $E_{n}$, состояшие из функционалов вида $\sum_{|\kappa|=n} c_{\kappa} \partial^{\kappa} \delta(x)$, инвариантны относительно $L_{+}^{\uparrow}$. Поэтому задача сводится к проверке того, что внутри каждого $E_{n}$ оператор $\mathrm{N}_{1}$ отображает подпространство $F_{n}=\bigcap_{i<j} \mathrm{Ker}_{i j}$ на подпространство, определяемое формулами (22), которое мы обозначим $G_{n}$. Преобразование Фурье переводит $E_{n}$ в пространство однородных многочленов степени $n$, которое мы разложим в прямую сумму $S O(3)$-инвариантных подпространств,

$$
E_{n}=\bigoplus_{l=0}^{n} p_{0}^{n-l} P_{l}
$$

где $P_{l}$ состоит из однородных многочленов степени $l$ от переменных $p_{1}, p_{2}, p_{3}$ (и где $\left.P_{0}=\mathbb{C}\right)$. Напомним [38], что каждое подпространство $P_{l}$, в свою очередь, является прямой суммой минимальных инвариантных подпространств вида $\left(\mathbf{p}^{2}\right)^{k} H_{l-2 k}, \quad k=$ $0,1, \ldots,[l / 2]$, где $H_{l-2 k}$ состоит из гармонических (т.е. удовлетворяюших уравнению Лапласа) однородных полиномов. На $H_{l-2 k}$ оператор Казимира кратен единичному и соответствуюшее собственное значение равно 2 лишь при степени однородности $l-2 k=1$. Далее, лишь кратные $p_{1}$ элементы $H_{1}$ удовлетворяют первому из условий (22). Следовательно, многочлены

$$
p_{0}^{n-2 k-1}\left(\mathbf{p}^{2}\right)^{k} p_{1}, \quad k=0,1, \ldots,\left[\frac{n-1}{2}\right],
$$

образуют базис в $G_{n}$. Базис в $F_{n}$ формируют многочлены

$$
p_{0}^{n-2 k}\left(\mathbf{p}^{2}\right)^{k}, \quad k=0,1, \ldots,\left[\frac{n}{2}\right] .
$$

Таким образом, $\operatorname{dim} F_{n} \geqslant \operatorname{dim} G_{n}$ и рассматриваемое отображение $F_{n} \rightarrow G_{n}$ действительно сюръективно. Наличие у него одномерного ядра при четных $n$ соответствует очевидной неоднозначности искомого разложения, связанной с возможностью добавления слагаемых вида $\sum_{l} c_{l} \square^{l} \delta(x)$. Остается показать, что решение уравнения (20) можно выбрать с соблюдением указанного в (17) ограничения на коэффициенты. Сузим при четных $n$ отображение $F_{n} \rightarrow G_{n}$ на линейную оболочку первых $(n / 2-1)$ из многочленов (25), что конретизирует выбор решения $v_{0}$. Применяя к ним генератор $p_{1} \partial_{0}+p_{0} \partial_{1}$, мы видим, что тогда при любом $n$ матрица $\left(a_{k l}\right)$ отображения в указанных базисах квазидиагональна и ее ненулевыми элементами являются лишь $a_{k k}=n-2 k$ и 
$a_{k, k+1}=2(k+1), 0 \leqslant k \leqslant[(n-1) / 2]$. Обратная матрица также верхнетреугольна, абсолютная величина ее элементов монотонно растет при одновременном увеличении обоих индексов и максимальна при $l=[(n-1) / 2]$, когда

$$
\left|a_{k l}^{(-1)}\right|=\frac{(n-1) ! !}{(n-2 k) ! !(2 k) ! !}
$$

в случае нечетных $n$. При четных $n$ числитель в формуле (26) заменяется на $(n-2) ! !$. Произведение левой и правой частей неравенства

$$
\frac{(n-1) ! !}{(n-2 k) ! !(2 k) ! !} \leqslant \frac{n ! !}{(n-2 k-1) ! !(2 k-1) ! !}
$$

есть биномиальный коэффициент $\left(\begin{array}{c}n \\ 2 k\end{array}\right)$, мажорируемый $2^{n}$. Таким образом, $\left|a_{k l}^{(-1)}\right| \leqslant$ $2^{n / 2}$. Далее, коэффициенты разложения элементов $G_{n}$ по базису (24) являются подмножеством коэффициентов разложения по мономам $p^{\kappa}$, а коэффициенты разложения элементов $F_{n}$ по мономам отличаются от коэффициентов разложения по базису (25) множителями, которые не превосходят $3^{n / 2}$. Таким образом, если $u=\sum_{\kappa} c_{\kappa} \partial^{\kappa} \delta(x)$, то для коэффициентов $c_{\kappa}^{0}$ указанного решения $v_{0}$ уравнения (20) справедлива оценка $\max _{|\kappa|=n}\left|c_{\kappa}^{0}\right| \leqslant 6^{n / 2} \max _{|\kappa|=n}\left|c_{\kappa}\right|$, что завершает доказательство теоремы 6 .

ЗАмЕчАниЕ. Изложенное доказательство прямо переносится на случай пространства-времени любой размерности $d \geqslant 3$. Однако при $d=2$, когда подгруппы врашений нет, оно утрачивает силу и аналогичная теорема не соблюдается.

Действительно, рассмотрим распределение $\partial_{+}\left[\theta\left(x_{+}\right) \ln x_{+}\right] \delta\left(x_{-}\right) \in S^{\prime}\left(\mathbb{R}^{2}\right)$, где $x_{ \pm}=$ $\left(x_{0} \pm x_{1}\right) / \sqrt{2}$ - конусные координаты. Оно не является лоренц-инвариантным в $\mathbb{R}^{2}$, имеет носителем луч на поверхности конуса $\overline{\mathbb{V}}_{+}^{(2)}$ и удовлетворяет уравнению $\mathrm{N}_{1} v=\delta(x)$ (в этих переменных $\left.\mathrm{N}_{1}=x_{+} \partial_{+}-x_{-} \partial_{-}\right)$. Этому же уравнению удовлетворяет распределение, получаемое из него операцией отражения $x \rightarrow-x$, однако среди функционалов (17) решений у данного уравнения нет. Поэтому сумма

$$
\partial_{+}\left[\theta\left(x_{+}\right) \ln x_{+}\right] \delta\left(x_{-}\right)+\partial_{+}\left[\theta\left(-x_{+}\right) \ln \left|x_{+}\right|\right] \delta\left(x_{-}\right)
$$

является нечетным лоренш-инвариантным распределением, которое не допускает инвариантного разложения ни в классе Шварца $S^{\prime}$, ни в классах $S^{\prime \beta}, \beta \geqslant 0$. Этот пример показывает также, что не любое лоренц-инвариантное распределение в $\mathbb{R}^{2} \backslash\{0\}$ допускает инвариантное продолжение на все пространство $\mathbb{R}^{2}$, в то время как в $\mathbb{R}^{4}$ такое продолжение всегда сушествует ([2], предложение 3.5$)$. С невозможностью продолжения распределения $\theta\left(x_{+}\right) x_{+}^{-1} \delta\left(x_{-}\right)$в $\mathbb{R}^{2} \backslash\{0\}$ до лоренш-инвариантной положительной меры на $\mathbb{R}^{2}$ связана необходимость использования индефинитной метрики при квантовании безмассового скалярного поля в двумерном пространстве-времени [39].

ТЕОРема 7. Пусть $v$-лоренц-инвариантный функционал класса $S^{\prime \beta}, \quad \beta \geqslant 0$, с несущим конусом $\overline{\mathbb{V}}$. Если его фурье-образ обращается в нуль в окрестности какой-либо пространственноподобной точки, то $v$ является нечетным. 
ДокАЗАТЕЛЬСТво. Пусть $v=v_{+}-v_{-}-$инвариантное разложение $v$ на функционалы с несушими конусами $\overline{\mathbb{V}}_{+}$и $\overline{\mathbb{V}}_{-}$. Теорема 4 из статьи [40] утверждает, что для $v_{ \pm}$ корректно определено преобразование Лапласа $\mathbf{u}_{ \pm}(\zeta)=\left(v_{ \pm}, e^{i(\cdot, \zeta)}\right)$. Функции $\mathbf{u}_{ \pm}$аналитичны в областях $\mathbb{T}_{ \pm}=\left\{\zeta=p+i \eta: \eta \in \mathbb{V}_{ \pm}\right\}$, и их граничными значениями при $\eta \rightarrow 0, \eta \in V_{ \pm}^{\prime}\left(\right.$ где $\left.\bar{V} I_{ \pm} \backslash\{0\} \subset \mathbb{V}_{ \pm}\right)$служат фурье-образы $u_{ \pm}=\mathcal{F} v_{ \pm}$. В статье [40] рассматривались классы $S_{\alpha}^{\prime 0} \supset S^{\prime \beta}$ и доказывалась сходимость к граничным значениям по топологии $S_{0}^{\prime \alpha}=\mathcal{F}\left(S_{\alpha}^{\prime 0}\right)$, но в данном случае она соблюдается, конечно, и по топологии $S_{\beta}^{\prime}=\mathcal{F}\left(S^{\prime \beta}\right)$, что устанавливается той же аргументацией. Согласно теореме Баргмана-Холла-Вайтмана [1], [2] каждая из лоренш-инвариантных функций $\mathbf{u}_{ \pm}$допускает симметричное относительно комплексной группы Лоренца $L_{+}(\mathbb{C})$ и, в частности, относительно полного отражения $\zeta \rightarrow-\zeta$ аналитическое продолжение в расширенную область $\mathbb{T}^{\mathrm{ext}}$, которая содержит все пространственноподобные точки. Учитывая свойства единственности аналитических функций $([41], \S 6)$, заключаем, что сделанное предположение о носителе $u=\mathcal{F} v$ влечет равенство $\mathbf{u}_{+}(\zeta)=\mathbf{u}_{-}(\zeta), \zeta \in \mathbb{T}^{\text {ext. }}$. Следовательно, для любой пробной функции $g \in S_{\beta}=\mathcal{F}\left(S^{\beta}\right)$ имеем

$$
\begin{aligned}
(u, g) & =\lim _{\eta \rightarrow 0, \eta \in V_{+}^{\prime}} \int\left(u_{+}(p+i \eta)-u_{-}(p-i \eta)\right) g(p) \mathrm{d} p= \\
& =\lim _{\eta \rightarrow 0, \eta \in V_{+}^{\prime}} \int\left(u_{-}(-p-i \eta)-u_{+}(-p+i \eta)\right) g(p) \mathrm{d} p=-(u, g(-\cdot)),
\end{aligned}
$$

что и требовалось доказать.

Отметим, что теорему 7 можно несколько усилить: в ее формулировке достаточно предполагать, что у функционала $v$ имеется замкнутый несуший конус $K \supset \mathbb{V}$, отличный от всего пространства. Тогда и $\overline{\mathbb{V}}$ будет его несушим конусом ввиду сушествования среди замкнутых несущих конусов наименьшего и вследствие лоренц-инвариантности $v$, поскольку любое пространственноподобное направление можно перевести внутрь дополнения к $K$ подходяшим преобразованием Лоренца.

\section{7. ТЕОРЕМА О ПЛОТНОСТИ}

Пусть $\mathcal{E}$ - конечномерное комплексное векторное пространство, в котором задано представление $T$ группы $L_{+}^{\uparrow}$. Обозначим через $L\left(S^{\beta}, \mathcal{E}\right)$ пространство непрерывных линейных отображений $S^{\beta}$ в $\mathcal{E}$, снабженное топологией равномерной сходимости на ограниченных множествах. Задав базис в $\mathcal{E}$, отображение $w \in L\left(S^{\beta}, \mathcal{E}\right)$ можно отождествить с набором непрерывных линейных функционалов $w^{j} \in S^{\prime \beta}$, число которых равно размерности представления (или, эквивалентным образом, с элементом пространства $\left.\left(\mathcal{E}^{\prime} \otimes S^{\beta}\right)^{\prime}\right)$. Поскольку $S^{\beta}$ - монтелевское пространство, сходимость последовательности $w_{\nu}$ по указанной топологии равносильна тому, что каждая из последовательностей $w_{\nu}^{j}$ слабо сходится. Отображение $w \in L\left(S^{\beta}, \mathcal{E}\right)$ называется (векторнозначной) лоренц-ковариантной обобщенной функцией, если оно удовлетворяет условию

$$
w(f)=T(\Lambda) w\left(f_{\Lambda}\right), \quad \Lambda \in L_{+}^{\uparrow}, \quad f \in S^{\beta},
$$


где действие группы Лоренца в пространстве пробных функций определяется обычным образом как $f_{\Lambda}(x)=f\left(\Lambda^{-1} x\right)$. Если $T$ имеет нечетную валентность и реализует представление группы $S L(2, \mathbb{C})$, являюшейся универсальной накрывающей для $L_{+}^{\uparrow}$, то аналогичное (28) требование приводит к тождественному равенству $w \equiv 0$, поэтому фактически речь идет об однозначных представлениях группы Лоренца. В компонентной записи условие (28) принимает вид

$$
\left(w^{j}, f_{\Lambda^{-1}}\right)=\sum_{k} T_{k}^{j}(\Lambda)\left(w^{k}, f\right), \quad \Lambda \in L_{+}^{\uparrow}, \quad f \in S^{\beta} .
$$

Лоренц-ковариантные обобщенные функции образуют замкнутое подпространство в $L\left(S^{\beta}, \mathcal{E}\right)$, которое мы снабжаем индуцированной топологией.

ТЕОРема 8. Пространство лорени-ковариантных распределений умеренного роста плотно в пространстве преобразующихся по тому же представлению группы Лоренца ковариантных обобщенных функций класса $S^{\prime \beta}$ при любом $\beta \geqslant 0$.

ДокАЗАТЕЛЬСтво. Регуляризуем ультрафиолетовое поведение ковариантной обобщенной функции $w$ умножением ее фурье-образа $u=\mathcal{F} w$ на $\chi(p / M)=\chi_{0}\left(p^{2} / M^{2}\right)$, где $p^{2}$ - лоренцев квадрат вектора $p$ и $\chi_{0}(t)$ - бесконечно дифференцируемая функция с носителем в интервале $(-1,1)$, тождественно равная 1 при $|t| \leqslant 1 / 2$. Пространство $S_{\beta}$ состоит из гладких функций $g$ таких, что

$$
\|g\|_{B, N}=\max _{\kappa \leqslant N} \sup _{p}\left|\partial^{\kappa} g(p)\right| \exp \left(\left|\frac{p}{B}\right|^{\frac{1}{\beta}}\right)<\infty
$$

при некотором $B$ (зависящем от $g$ ) и любом $N=0,1, \ldots$ Поэтому очевидно, что $\chi$ является мультипликатором в $S_{\beta}$, а с учетом оценки

$$
\left|\partial^{\kappa} \chi\left(\frac{p}{M}\right)\right| \leqslant C_{\kappa}\left|\frac{p}{M^{2}}\right|^{|\kappa|}
$$

легко проверяется, что $g \chi(p / M) \rightarrow g$ в $S_{\beta}$ при $M \rightarrow \infty$. Следовательно, $u_{M}^{j}=$ $u^{j} \chi(p / M) \rightarrow u^{j}$ в $S_{\beta}^{\prime}$. Остается показать, что функционалы $u_{M}^{j}$ допускают непрерывное продолжение на пространство Шварца $S$. Поскольку $S_{\beta}^{\prime} \subset S_{0}^{\prime}=\mathcal{D}^{\prime}$, то можно воспользоваться критерием, указанным в книге [42] (§II.10.7), который гласит, что распределение из $\mathcal{D}^{\prime}$ продолжается на $S$ в том и только в том случае, если его свертка с любой пробной функцией $g \in \mathcal{D}$, имеющей носитель в единичном шаре $|p|<1$, растет на бесконечности не быстрее, чем степенным образом. Рассмотрим сначала случай лоренц-инвариантных функционалов. Значение свертки $\left(u_{M} * g\right)$ в точке $q$ есть значение распределения $u_{M}$ на сдвинутой функции $g(p-q)$. Нуждаются в рассмотрении лиш сдвиги по поверхности светового конуса, ибо для остальных направлений $\left(u_{M} * g\right)(q)$ обращается в нуль при достаточно больших $|q|$. Кроме того, можно считать, что $q_{2}=q_{3}=0$, поскольку любой вектор $q^{\prime} \in \mathbb{R}^{4}$ переводится в некоторую точку $q$ этой плоскости подходящим пространственным поворотом $R$ и $\left(u_{M} * g\right)\left(q^{\prime}\right)=\left(u_{M} *\right.$ $\left.g_{R}\right)(q)$, где $g_{R}(\cdot)=g\left(R^{-1}(\cdot)\right)$. Наконец, не ограничивая обшности, можно положить 
$M=1$, так как $S^{\prime}$ и $S_{\beta}^{\prime}$ инвариантны относительно гомотетий. Воспользуемся конусными переменными $q_{ \pm}=\left(q_{0} \pm q_{1}\right) / \sqrt{2}$, и пусть, для определенности, $q_{-}=0, q_{+} \rightarrow+\infty$. Обозначим через $\Lambda$ преобразование Лоренца $p_{+} \rightarrow p_{+} / q_{+}, p_{-} \rightarrow q_{+} p_{-}$в плоскости $\left(p_{0}, p_{1}\right)$, которое переводит $q$ в вектор с единичной евклидовой нормой. Ввиду лоренщ-инвариантности $u$ и $\chi$ имеем

$$
(u \chi * g)(q)=\left(u, g_{q}\right), \quad \text { где } \quad g_{q}(p)=\chi(p) g\left(q-\Lambda^{-1} p\right) .
$$

Точки $\operatorname{supp} g_{q}$ по построению удовлетворяют неравенствам $\left|p^{2}\right|<1, p_{2}^{2}+p_{3}^{2}<1$, и поэтому $\left|p_{+} p_{-}\right|<1$. Кроме того $\left|q_{+}-q_{+} p_{+}\right|<1$ и, как следствие, $\left|p_{-}\right|<1 /\left(1-1 / q_{+}\right)$. Значит, если $q_{+}$достаточно велико, то $\operatorname{supp} g_{q}$ содержится в шаре радиуса 2 и справедлива оценка

$$
\left|\left(u, g_{q}\right)\right| \leqslant\|u\|_{2, N}\left\|g_{q}\right\|_{2, N},
$$

где $\left\|g_{q}\right\|_{2, N}=\max _{|\kappa| \leqslant N} \sup _{|p| \leqslant 2}\left|\partial^{\kappa} g_{q}(p)\right|$ в соответствии с формулой (30) и $N$ имеет смысл порядка сингулярности распределения $u$ в шаре $|p|<2$. Преобразование $\Lambda^{-1}$ производит сжатие графика функции $g$ в $q_{+}$раз по переменной $p_{+}$, поэтому

$$
\sup _{p}\left|\partial^{\kappa} g\left(q-\Lambda^{-1} p\right)\right|=\sup _{p}\left|\partial^{\kappa} g\left(\Lambda^{-1} p\right)\right| \leqslant C_{\kappa}\|g\|_{1, N} q_{+}^{|\kappa|}, \quad|\kappa| \leqslant N
$$

что вместе с оценкой (31) дает

$$
\left\|g_{q}\right\|_{2, N} \leqslant C_{N}\|g\|_{1, N}(1+|q|)^{N} .
$$

Заключаем, что поведение $\left(u_{M} * g\right)(q)$ при $|q| \rightarrow \infty$ действительно не хуже степенного (со степенью, зависящей от $M$ ). В случае лоренц-ковариантной обобщенной функции оценку $\left(u_{M}^{j} * g\right)(q)$ можно выполнить аналогичным образом, используя правило преобразования (29), что приводит к такому же заключению, поскольку при этом матричные элементы представления $T_{k}^{j}(\Lambda)$ являются рациональными функциями параметра буста $q_{+} \cdot$ Теорема 8 доказана.

\section{8. РАЗЛОЖКЕНИЕ ПО ПОЛИНОМИАЛЬНЫМ КОВАРИАНТАМ}

Будем использовать обозначение $(r, s)$, где $r, s$ - неотрицательные целые или полуцелые числа, для неприводимых конечномерных представлений группы $S L(2, \mathbb{C})$ и реализуем их обычным образом в пространствах комплексных однородных полиномов от спинорных переменных $\omega=\left(\omega_{1}, \omega_{2}\right)$ и $\bar{\omega}=\left(\bar{\omega}_{1}, \bar{\omega}_{2}\right)$ степени $2 r$ и $2 s$, соответственно. Напомним, что стандартный полиномиальный ковариант, преобразующийся по представлению $(s, s)$, имеет вид $(\bar{\omega} \tilde{x} \omega)^{2 s}$, где

$$
\tilde{x}=\left(\begin{array}{cc}
x_{0}-x_{3} & -x_{1}+i x_{2} \\
x_{1}+i x_{2} & x_{0}+x_{3}
\end{array}\right) .
$$

Покажем, что представление [2], [23] лоренц-ковариантных распределений умеренного роста через полиномиальные коварианты допускает обобшение на функционалы класса $S^{\prime \beta}$. Векторная обобшенная функция $w$ теперь трактуется как комплекснозначная обобщенная функция по переменной $x$, которая зависит также от переменных $\omega, \bar{\omega}$ полиномиальным образом, и условие лоренц-ковариантности (28) принимает вид

$$
w(x ; \omega, \bar{\omega})=w(\Lambda(A) x ; A \omega, \bar{A} \bar{\omega}), \quad A \in S L(2, \mathbb{C}),
$$

где $A \rightarrow \Lambda(A)$ - канонический гомоморфизм группы $S L(2, \mathbb{C})$ в $L_{+}^{\uparrow}$. 
ТЕОРема 9. Лорени-ковариантная обобщенная функиия $w$, определенная на пространстве $S^{\beta}, \quad \beta \geqslant 0$, и преобразующаяся по представлению $(r, s)$, отлична от нуля лишь при $r=s ;$ в этом случае она представима в виде

$$
w(x ; \omega, \bar{\omega})=(\bar{\omega} \tilde{x} \omega)^{2 s} v(x),
$$

где $v \in S^{\prime \beta}$ - лорени-инвариантный функционал, определяемый по $w$ с точностью до слагаемого $\sum_{l=0}^{2 s-1} c_{l} \square^{l} \delta(x)$, включающего произвольные постоянные $c_{l}$.

(Отметим, что $(s, s)$ является однозначным представлением группы $L_{+}^{\uparrow}$.)

Для доказательства этой теоремы потребуются две леммы.

Лемма 2. Пусть $f \in S^{\beta}\left(\mathbb{R}^{n}\right), \quad \beta \geqslant 0$. Eсли $\left.f\right|_{x_{1}=0}=0$, mo $f(x)=x_{1} f_{1}(x)$, әде функиия $f_{1}$ также принадлежит $S^{\beta}\left(\mathbb{R}^{n}\right)$.

ДоКАЗАТЕЛЬСТво. Будем использовать обозначение $x^{\prime}=\left(x_{2}, \ldots, x_{n}\right)$ и положим

$$
f_{1}\left(x_{1}, x^{\prime}\right)=\int_{0}^{1}\left(\partial_{1} f\right)\left(t x_{1}, x^{\prime}\right) d t .
$$

При $\left|x_{1}\right| \leqslant 1$ имеем оценку

$$
\begin{aligned}
\left|\partial^{\kappa} f_{1}(x)\right| & \leqslant\|f\|_{B, N} B^{|\kappa|+1} \frac{\left(\kappa_{1}+1\right)^{\beta\left(\kappa_{1}+1\right)}}{\kappa_{1}^{\beta \kappa_{1}}} \kappa^{\beta \kappa}\left(1+\left|x^{\prime}\right|\right)^{-N} \leqslant \\
& \leqslant C_{\epsilon, N}\|f\|_{B, N}(B+\epsilon)^{|\kappa|} \kappa^{\beta \kappa}(1+|x|)^{-N}
\end{aligned}
$$

где норма определяется формулой (2) и число $\epsilon>0$ сколь угодно мало. При $\left|x_{1}\right|>1$ прямое применение к $f_{1}=f / x_{1}$ формулы Лейбница дает

$$
\left|\partial^{\kappa} f_{1}(x)\right| \leqslant\|f\|_{B, N} \sum_{\ell \leqslant \kappa}\left(\begin{array}{c}
\kappa \\
\ell
\end{array}\right) B^{|\kappa-\ell|}(\kappa-\ell)^{\beta(\kappa-\ell)} \ell !(1+|x|)^{-N} .
$$

Если $\beta>1$, то $\ell ! \leqslant C_{\epsilon} \epsilon^{|\ell|} \ell^{\beta \ell}$ и, учитывая неравенство $(\kappa-\ell)^{\beta(\kappa-\ell)} \ell^{\beta \ell} \leqslant \kappa^{\beta \kappa}$, заключаем, что и в этой области для функции $f_{1}$ справедлива оценка типа (40), т.е. $f_{1} \in S^{\beta}$. Пусть теперь $\beta=1$. Пространство $S^{1}$ представимо как объединение по $B$ пространств функций, аналитических в областях $T^{B}=\left\{z=x+i y \in \mathbb{C}^{n}:\left|y_{j}\right|<1 / B, \quad \forall j\right\}$ и имеющих конечные нормы

$$
\|f\|_{B, N}=\sup _{z \in T^{B}}|f(z)|(1+|x|)^{N}, \quad N=1,2, \ldots
$$

Из условия $\left.f\right|_{z_{1}=0}=0$ следует, что $f(z)=z_{1} f_{1}(z)$, где $f_{1}$ аналитична в той же трубе, что и функция $f$, и мажорируется ею при $\left|z_{1}\right| \geqslant \delta>0$. Для оценки $f_{1}(z)$ при $\left|z_{1}\right|<\delta$ воспользуемся формулой Коши по переменной $z_{1}$, полагая $\delta=1 /(3 B)$ и взяв в качестве контура интегрирования окружность радиуса $2 \delta$. Такой контур лежит в $T^{B}$ и $\left|\zeta_{1}\right|>\delta$ для любой точки $\zeta$ на нем. В результате получаем

$$
\left|f_{1}(z)\right| \leqslant C_{\delta, N}\|f\|_{B, N}(1+|x|)^{-N} .
$$


При $\beta<1$ оценка выполняется аналогично. Функция $f_{1}(z)$ в этом случае является целой, нормами служат выражения

$$
\sup _{z}|f(z)|(1+|x|)^{N} \exp \left(-|B y|^{\frac{1}{1-\beta}}\right),
$$

а в качестве $\delta$ можно взять 1 . Тем самым лемма 2 доказана. Отметим, что наличие норм функции $f$ в правых частях оценок демонстрирует секвенциальную непрерывность обратного к инъективному отображению $f \rightarrow x_{1} f$ пространства $S^{\beta}$ в себя.

ЛЕмма 3. Любая функиия $f \in S^{\beta}\left(\mathbb{R}^{n}\right), \quad \beta \geqslant 0$, удовлетворяющая условию $\partial^{\kappa} f(0)=0$ при всех $|\kappa| \leqslant m n$, допускает разложсение вида

$$
f(x)=\sum_{i=1}^{n} x_{i}^{m+1} f_{i}(x), \quad \text { əде } f_{i} \in S^{\beta}\left(\mathbb{R}^{n}\right) .
$$

ДоКАЗАТЕЛЬСТво. При $n=1$ справедливость такого представления прямо следует из леммы 2. Далее воспользуемся индукцией по $n$, вводя обозначения

$$
g_{j}\left(x^{\prime}\right)=\left.\frac{1}{j !} \partial_{1}^{j} f(x)\right|_{x_{1}=0}, \quad F(x)=f(x)-f_{0}\left(x_{1}\right) \sum_{j=0}^{m} x_{1}^{j} g_{j}\left(x^{\prime}\right),
$$

где $x^{\prime}=\left(x_{2}, \ldots, x_{n}\right)$, как и прежде, и где функция $f_{0} \in S^{\beta}(\mathbb{R})$ подчинена условиям $f_{0}^{(j)}(0)=0,0 \leqslant j \leqslant m$. При всех $|\kappa| \leqslant m(n-1)$ имеем $\partial^{\kappa} g_{j}(0)=0$ и по предположению индукции

$$
g_{j}\left(x^{\prime}\right)=\sum_{i=2}^{n} x_{i}^{m+1} f_{i j}\left(x^{\prime}\right), \quad \text { где } \quad f_{i j} \in S^{\beta}\left(\mathbb{R}^{n-1}\right) .
$$

Далее, $\left.\partial_{1}^{j} F\right|_{x_{1}=0}=0$ при всех $j \leqslant m$. Значит, $F(x)=x_{1}^{m+1} f_{1}\left(x^{\prime}\right)$ в силу леммы 2 и равенство (45) соблюдается с функцией $f_{i}(x)=f_{0}\left(x_{1}\right) \sum_{j=0}^{m} x_{1}^{j} f_{i j}\left(x^{\prime}\right), 2 \leqslant i \leqslant n$.

ДОКАЗАТЕЛЬСТво ТЕОРЕМЫ 9 . Тождественное равенство $w$ нулю при $r \neq s$ следует из теоремы 8 и из предложения 3.6 книги [2] о структуре лоренц-ковариантных распределений Шварца. Размерность представления $(s, s)$, т.е. число различных мономов от переменных $\omega, \bar{\omega}$ степени $2 s$ по каждой из них, равна $(2 s+1)^{2}$. Перенумеруем эти мономы и рассмотрим отображение

$$
h: \bigoplus^{(2 s+1)^{2}} S^{\beta} \rightarrow S^{\beta}
$$

сопоставляющее набору пробных функций $\left\{f_{i}\right\}, 1 \leqslant i \leqslant(2 s+1)^{2}$, их линейную комбинацию, получаемую заменой мономов в многочлене $(\bar{\omega} \tilde{x} \omega)^{2 s}$ на пробные функции с соответствующими индексами. Сопряженное к $h$ отображение $h^{\prime}$ сопоставляет функционалу $v \in S^{\prime \beta}$ набор функционалов, получаемых из $v$ умножением на коэффициенты многочлена, и сужение $h^{\prime}$ на подпространство инвариантных функционалов переводит их в ковариантные функционалы вида (38). Поскольку любое ковариантное распределение 
Шварца имеет такой вид и они плотны в ковариантных обобщенных функциях рассматриваемого класса, достаточно показать, что образ любого замкнутого подпространства при отображении $h^{\prime}$ замкнут в $\bigoplus^{(2 s+1)^{2}} S^{\prime \beta}$.

Заметим, что $\operatorname{Im} h$ включает в себя замкнутое подпространство, выделяемое условиями $\partial^{\kappa} f(0)=0,|\kappa| \leqslant 4 s(4 s-1)$, которое имеет конечную коразмерность в $S^{\beta}$. Действительно, согласно лемме 3 имеется разложение $f(x)=\sum_{i=0}^{3} x_{i}^{4 s} f_{i}(x)$, и если выразить $x_{i}^{4 s}$ через элементы $x_{\rho \sigma}$ матрицы (36), то каждый член получающегося выражения будет содержать хотя бы один из этих элементов в степени не меньшей $2 s$. Значит, функция $f$ может быть записана как сумма

$$
\sum_{\rho, \sigma=1,2} x_{\rho \sigma}^{2 s} f_{\rho \sigma} \quad\left(f_{\rho \sigma} \in S^{\beta}\right),
$$

которая, очевидно, содержится в $\operatorname{Im} h$. Таким образом, подпространство $\operatorname{Im} h$ представимо как сумма конечномерного и замкнутого подпространств и, следовательно, тоже замкнуто. Далее, благодаря рефлексивности $S^{\beta}$ из замкнутости $\operatorname{Im} h$ вытекает, что отображение $h^{\prime}$ является гомоморфизмом (открытым отображением на свой образ) относительно сильных топологий сопряженных пространств [43] (§ IV.4.1). Пусть $L$ - замкнутое подпространство в $S^{\prime \beta}$. Его сумма с конечномерным подпространством Ker $h^{\prime}$ (которое содержится в линейной оболочке функционалов $\partial^{\kappa} \delta(x),|\kappa| \leqslant 4 s(4 s-1)$ ) также замкнута. Поэтому для точки $v \notin L+\operatorname{Ker} h^{\prime}$ найдется окрестность $\mathcal{U}$, которая не пересекается с $L+\operatorname{Ker} h^{\prime}$. Множество $h^{\prime}(\mathcal{U})$ является окрестностью в $\operatorname{Im} h^{\prime}$ и не пересекается с подпространством $h^{\prime}(L)$. Следовательно, $h^{\prime}(L)$ замкнуто, что и требовалось доказать.

Последнее утверждение теоремы уточняет описание ядра ограничения оператора $h^{\prime}$ на подпространство лоренц-инвариантных функционалов. Поскольку инвариантные комбинации распределений $\partial^{\kappa} \delta(x)$ имеют вид $\sum_{l} c_{l} \square^{l} \delta(x)$ и при преобразовании Фурье переходят в полиномы по $p^{2}$, а фурье-образом $(\bar{\omega} \tilde{x} \omega)$ служит $-i(\bar{\omega} \tilde{\partial} \omega)$, то доказательство завершается применением тождеств $(\bar{\omega} \tilde{\partial} \omega)\left(p^{2}\right)=2(\bar{\omega} \tilde{p} \omega),(\bar{\omega} \tilde{\partial} \omega)(\bar{\omega} \tilde{p} \omega)=0$. Отсюда следует, что $(\bar{\omega} \tilde{\partial} \omega)^{2 s}\left(p^{2}\right)^{l}=0$ лишь при $l \leqslant 2 s-1$. Теорема 9 доказана.

Теперь мы в состоянии распространить теорему 6 на ковариантные обобщенные функции, но для этого понадобится еще одно вспомогательное утверждение, которое примыкает к лемме 2.

Лемма 4. Пусть $v \in S^{\prime \beta}, \beta \leqslant 1$, и пусть $K-$ замкнутый конус в $\mathbb{R}^{n}$, в котором содержится плоскость $x_{1}=0$. Если $K$ является несущим конусом для $x_{1} v$, то он будет несущим и для $v$.

ДокаЗАТЕЛЬСтво. Пусть $O$ - объединение открытого конуса $U \supset K \backslash\{0\}$ с $\epsilon$-окрестностью начала координат и пусть $f \in S^{\beta}(O)$. Обозначим $g\left(z^{\prime}\right)=f\left(0, z^{\prime}\right)$. Поскольку точки вида $\left(0, z^{\prime}\right)$ лежат в $O$, функция $g$ принадлежит $S^{\beta}\left(\mathbb{R}^{n-1}\right)$ и

$$
\|g\|_{B, N} \leqslant\|f\|_{O, B, N} .
$$

При $\beta=1$ определение нормы $\|f\|_{O, B, N}$ отличается от (42) тем, что sup берется по комплексной $(1 / B)$-окрестности $O$, а при $\beta<1$ норма включает дополнительный к (44) 
множитель $\exp \left\{-d(B x, U)^{1 /(1-\beta)}\right\}$ в соответствии с формулой (13). Пусть теперь $f_{0}\left(z_{1}\right)$ - любая функция из $S^{\beta}(\mathbb{R})$, равная 1 в нуле. Тогда $\left(f-f_{0} g\right) \in S^{\beta}(O)$ и $(f-$ $\left.f_{0} g\right)\left.\right|_{z_{1}=0}=0$. Те же элементарные соображения, что и при доказательстве леммы 2 , показывают, что $\left(f-f_{0} g\right)(z)=z_{1} f_{1}(z)$, где $f_{1}$ принадлежит пространству $S^{\beta}(O)$ и стремится в нем к нулю, когда $f$ стремится к нулю по его топологии. Таким образом, формула

$$
(\hat{v}, f)=\left(v, f_{0} g\right)+\left(x_{1} v, f_{1}\right)
$$

определяет непрерывное продолжение функционала $v$ на $S^{\beta}(O)$, что и доказывает утверждение леммы. Аналогичное утверждение верно и при $\beta>1$, но в этом случае оно тривиально. Лемма 4 доказана.

Теорема 10. Любая лоренц-ковариантная обобщенная функиия $w$ над $S^{\beta}, \beta \geqslant 0$, с несущим конусом $\overline{\mathbb{V}}$ допускает разложение на лорени-ковариантные обобщенные функции того же класса с несущими конусами $\overline{\mathbb{V}}_{+} u \overline{\mathbb{V}}_{-}$.

ДоКАЗАТЕЛЬСТво. Будем считать, что $w$ преобразуется по представлению $(s, s)$. Конус $\overline{\mathbb{V}}$ является несушим для инвариантного функционала $v$, через который функция $w$ выражается формулой (38). Действительно, по условию теоремы он служит несушим для $\left(x_{0}-x_{3}\right)^{2 s} v$. Считая разность $x_{0}-x_{3}$ первой координатой и применяя лемму 4 , заключаем, что дополнение к конической окрестности положительной $x_{3}$-полуоси заведомо является несушим конусом $v$, а в силу лоренц-инвариантности то же самое можно сказать и относительно любого другого пространственноподобного направления; пересечение же этих дополнительных конусов есть $\overline{\mathbb{V}}$. Остается учесть существование наименьшего несушего конуса и применить теорему 6 . Теорема 10 доказана.

\section{9. ПРИЛОЖЕНИЕ К ТЕОРЕМЕ О СВЯЗИ СПИНА СО СТАТИСТИКОЙ}

Рассмотрим конечную совокупность полей $\left\{\phi_{i}\right\}$, являющихся операторнозначными обобшенными функциями над пространством $S^{\beta}\left(\mathbb{R}^{4}\right), \beta<1$, и удовлетворяюших обычньм предположениям вайтмановской аксиоматики [1], [2] за исключением локальной коммутативности, формулировку которой использование аналитических пробных функций делает невозможной. Естественной заменой этой аксиомы, причем по своему смыслу более близкой к физическому требованию макропричинности, является условие, что замкнутый световой конус $\overline{\mathbb{V}}$ служит несушим конусом матричных элементов коммутаторов наблюдаемых полей. (Если теория включает и ненаблюдаемые поля, то $\overline{\mathbb{V}}$ является несушим конусом либо коммутаторов, либо антикоммутаторов.) Подробнее о мотивировке и точной формулировке этого условия, которое мы называем асимптотической коммутативностью, см. статью [24]. Мы придерживаемся обычного предположения, что тип перестановочного соотношения зависит лишь от типа поля и одинаков для всех его лоренцевых компонент, поэтому лоренцевы индексы в дальнейшем не выписываем. Из трансформационных свойств полей относительно группы Пуанкаре и инвариантности вакуума следует, что вакуумные средние $\left\langle\Psi_{0}, \phi\left(x_{1}\right) \psi\left(x_{2}\right) \Psi_{0}\right\rangle$ являются лоренш-ковариантными обобшенными функциями над $S^{\beta}\left(\mathbb{R}^{4}\right)$ по разностной переменной 
$\xi=x_{1}-x_{2}$. Это позволяет применить теорему 9 к обобшению вывода связи спина со статистикой на нелокальные поля. В полной аналогии с обычной теорией [1], [2] полей умеренного роста слабое кластерное свойство вакуумных средних (которое следует из существования и единственности вакуума без использования локальности) приводит к тому, что любая пара ненулевых полей $\phi, \psi$, определенных на $S^{\beta}$, имеет тот же тип перестановочных соотношений, что и пара $\phi, \psi^{*}$ (см. теорему 11 в [24]). Поэтому задача сводится к анализу асимптотических коммутационных соотношений между полем $\phi$ и эрмитово сопряженным ему полем $\phi^{*}$.

Теорема 11. Пусть поле $\phi$ определено на пространстве $S^{\beta}\left(\mathbb{R}^{4}\right), \quad 0 \leqslant \beta<1, u$ преобразуется по неприводимому представлению $(r, s)$ группь $S L(2, \mathbb{C})$. Аномальное асимптотическое перестановочное соотношение между $\phi и \phi^{*}$ (антикоммутативность в случае челого спина и коммутативность в случае полуцелого) приводит к равенству $\phi(f) \Psi_{0}=\phi^{*}(f) \Psi_{0}=0$ для всех $f \in S^{\beta}\left(\mathbb{R}^{4}\right)$.

ДОКАЗАТЕЛЬСТво. Будем использовать обозначения

$$
W\left(x_{1}-x_{2}\right)=\left\langle\Psi_{0}, \phi\left(x_{1}\right) \phi^{*}\left(x_{2}\right) \Psi_{0}\right\rangle, \quad W^{\prime}\left(x_{1}-x_{2}\right)=\left\langle\Psi_{0}, \phi^{*}\left(x_{1}\right) \phi\left(x_{2}\right) \Psi_{0}\right\rangle
$$

и рассмотрим сначала случай скалярного поля. Аномальное асимптотическое перестановочное соотношение означает, что конус $\overline{\mathbb{V}}$ является несушим для функционала $W(\xi)+W^{\prime}(-\xi)$. Тогда согласно теореме 7 этот функционал является нечетным, поскольку в силу спектрального условия его фурье-образ обращается в нуль при $p^{2}<0$. Значит, нечетен и функционал $W(\xi)+W^{\prime}(\xi)$, который в импульсном представлении имеет носитель в конусе $\overline{\mathbb{V}}_{+}$и в таком случае обязан быть тождественно равным нулю. Взяв его значение на пробной функции вида $\bar{f}\left(x_{1}\right) f\left(x_{2}\right)$, получаем

$$
\left\|\phi(f) \Psi_{0}\right\|^{2}+\left\|\phi^{*}(f) \Psi_{0}\right\|^{2}=0, \quad f \in S^{\beta} .
$$

Пусть теперь поле $\phi$ преобразуется по неприводимому представлению $(r, s)$. Тогда аномальное перестановочное соотношение означает, что $\overline{\mathbb{V}}$ является несущим конусом для $W(\xi) \pm W^{\prime}(-\xi)$, где знак плюс соответствует случаю целого спина, а знак минус случаю полуцелого. Лоренц-ковариантные обобшенные функции (50) преобразуются по представлению $(r, s) \otimes(s, r)$, разложение которого на неприводимые представления имеет вид

$$
(r, s) \otimes(s, r)=\bigoplus_{\substack{|r-s| \leqslant r^{\prime}, s^{\prime} \leqslant r+s \\ r^{\prime}, s^{\prime} \in|r-s|+\mathbb{N}}}\left(r^{\prime}, s^{\prime}\right) .
$$

Соответственно в разложении $W(\xi) \pm W^{\prime}(-\xi)$ по полиномиальным ковариантам будет $2 \min (r, s)+1$ слагаемых. Отметим, что в случае целого спина, когда $r+s-$ целое число, в разложении участвуют коварианты лишь четной степени, а в случае полуцелого лишь нечетной. Применим теорему 10 и совершим преобразование Лапласа. Тогда в импульсном пространстве обобщенная функция $\widetilde{W}(p) \pm \widetilde{W^{\prime}}(-p)$ будет представлена в виде разности граничных значений лоренш-ковариантных аналитических функций, голоморфных в трубах $\mathbb{T}_{ \pm}$и допускаюших аналитическое продолжение в расширенную 
область $\mathbb{T}^{\text {ext }}$ по теореме Баргмана-Холла-Вайтмана [1], [2]. Эти аналитические функции симметричны относительно полного отражения $p+i \eta \rightarrow-p-i \eta$ в случае целого спина и антисимметричны в случае полуцелого, а в силу спектрального условия и теоремы единственности совпадают друг с другом в $\mathbb{T}^{\mathrm{ext}}$. Для граничных значений отсюда следует тождество

$$
\widetilde{W}(p) \pm \widetilde{W^{\prime}}(-p)=\mp \widetilde{W}(-p)-\widetilde{W^{\prime}}(p)
$$

Снова учитывая спектральное условие, мы видим, что тогда носителем $\widetilde{W}(p)+\widetilde{W}^{\prime}(p)$ может быть только точка $p=0$. Порядок сингулярности этой обобшенной функции должен быть равен нулю в силу условия положительности. В случае полуцелого спина такого члена в ее разложении по ковариантам нет, а в случае целого спина он не может преобразовываться при отражении по формуле (53). Таким образом, $\widetilde{W}(p)+\widetilde{W}^{\prime}(p) \equiv 0$, что завершает доказательство.

\section{0. ЗАКЛЮЧИТЕЛЬНЫЕ ЗАМЕЧАНИЯ}

Полученные результаты позволяют обращаться с высокосингулярными лоренц-ковариантными обобщенными функциями столь же просто, как и с обычными распределениями умеренного роста. Сушественным дополнением теоремы 7 служат теорема 2.14 из работы [22] и теорема 9 из статьи [24], которые показывают, что конус $\overline{\mathbb{V}}$ является несущим конусом нечетных лоренц-инвариантных функционалов при любой сингулярности. В разделе 9 рассматривались двухточечные вайтмановские функции специального вида, однако естественный аналог ковариантного разложения [2], [23] имеется для вакуумного среднего любой пары полей над $S^{\beta}$, преобразуюшихся по конечномерным неприводимым или просто приводимым представлениям группы $S L(2, \mathbb{C}) . \mathrm{K}$ открытым вопросам относится вывод для четных и нечетных инвариантных функционалов класса $S^{\prime \beta}$ аналога представления Мете. Отметим еще, что полученные теоремы можно распространить на функционалы, определенные на обобщенных пространствах Гельфанда-Шилова $S^{b}$, задаваемых индикаторной функцией $b$, характеризующей рост их фурье-образов. Соответствуюшие ограничения на индикаторную функцию установлены в статье [21].

Благодарности. Автор благодарен Российскому фонду фундаментальных исследований за финансовую поддержку, оказанную грантом № 99-01-00376 и грантом поддержки ведуших научных школ № 00-15-96566.

\section{Список литературы}

[1] P. Стритер, A. Вайтман. РСТ, спин и статистика и все такое. М.: Наука, 1966.

[2] Н. Н. Боголюбов, А. А. Логунов, А. И. Оксак, И. Т. Тодоров. Общие принципы квантовой теории поля. М.: Наука, 1987.

[3] Н. Н. Мейман. ЖЭТФ. 1964. Т. 47. С. 1966.

[4] A. Jaffe. Phys. Rev. 1967. V. 158. P. 1454.

[5] М. З. Иофа, В. Я. Файнберг. ТМФ. 1967. Т. 1. С. 187.

[6] М.З. Иофа, В.Я. Файнберг. ЖЭТФ. 1969. Т. 56. С. 1644

[7] V. Ya. Fainberg, A. V. Marshakov. Phys. Lett. B. 1988. V. 211. P. 82.

[8] M.Z. Iofa, V. Ya. Fainberg. Nuovo Cim. 1971. V. 5A. P. 273.

7 Теоретическая и математическая физика, т. 128, № 3, 2001 г. 
[9] В.Я. Файнберг. О квантовых теориях с неполиномиальным ростом матричных элементов. В сб.: Проблемы теоретической физики. М.: Наука, 1972. С. 119.

[10] V. Ya. Fainberg, M. A. Soloviev. Ann. Phys. 1978. V. 113. P. 421.

[11] S. B. Giddings. Phys. Rev. D. 2000. V. 61. P. 106008.

[12] Г. В. Ефимов. Нелокальные взаимодействия квантованных полей. М.: Наука, 1977.

[13] Г. В. Ефимов. Проблемы квантовой теории нелокальных взаимодействий. М.: Наука, 1985.

[14] J.W. Moffat. Quantum field theory solution to the gauge hierarchy and cosmological constant problems. hep-ph/0003171.

[15] M. А. Соловьев. ТМФ. 1971. Т. 7. С. 183.

[16] S. Nagamachi, N. Mugibayashi. Commun. Math. Phys. 1976. V. 46. P. 119.

[17] S. Nagamachi, N. Mugibayashi. Commun. Math. Phys. 1976. V. 49. P. 257.

[18] М. А. Соловьев. ТМФ. 1973. Т. 15. С. 3.

[19] U. Moschella, F. Strocchi. Lett. Math. Phys. 1992. V. 24. P. 103.

[20] M. A. Soloviev. Lett. Math. Phys. 1997. V. 41. P. 265.

[21] А.Г. Смирнов, М. А. Соловьев. ТМФ. 2000. Т. 123. С. 355.

[22] М. А. Соловьев. Труды ФИАН. 1993. Т. 209. С. 121.

[23] A. I. Oksak, I. T. Todorov. Commun. Math. Phys. 1969. V. 14. P. 271.

[24] М. А. Соловвев. ТМФ. 1999. Т. 121. С. 139.

[25] И. М. Гельфанд, Г. Е. Шилов. Обобщенные функции. Т. 2. М.: Физмат, 1958.

[26] В. П. Паламодов. УМН. 1971. Т. 26. С. 3.

[27] Х. Шефер. Топологические векторные пространства. М.: Мир, 1971.

[28] И. М. Гельфанд, Н. Я. Виленкин. Обобщенные функции. Т. 4. М.: Физмат, 1961.

[29] Л. Хермандер. Анализ линейных дифференциальных операторов с частными производными. Т. 1. М.: Мир, 1986.

[30] A. Lambert. Ann. Inst. Fourier. 1979. V. 29. P. 57.

[31] П. Шапира. Теория гиперфункций. М.: Мир, 1972.

[32] T. Kawai. J. Fac. Sci. Univ. Tokyo. Sect. 1A. Math. 1970. V. 17. P. 467.

[33] Д. А. Райжов. Сиб. матем. ж. 1966. Т. 7. С. 353.

[34] A. Grothendieck. Mem. Amer. Math. Soc. 1955. V. 16.

[35] В. С. Реmax. ДАН. 1970. Т. 194. С. 1277.

[36] В.Я. Файнберг, М. А. Соловвев. ТМФ. 1992. Т. 93. С. 514.

[37] M. A. Soloviev. Lett. Math. Phys. 1995. V. 33. P. 49.

[38] Д. П. Желобенко. Компактные группы Ли и их представления. М.: Наука, 1970.

[39] A.S. Wightman. Adv. Math. Suppl. Stud. 1981. V. 7B. P. 769.

[40] M. A. Soloviev. Commun. Math. Phys. 1997. V. 184. P. 579.

[41] В. С. Владимиров. Методы теории функций многих комплексных переменных. М.: Наука, 1964.

[42] Г. Е. Шилов. Математический анализ. Второй специальный курс. М.: Наука, 1965.

[43] Н. Бурбаки. Топологические векторные пространства. М.: ИЛ, 1959. 